\title{
Interactive System for Study of Car's Movement with Low and Ultra-low Profile Tires in a Turn
}

\author{
Stiliyana Taneva \\ Technical University - Sofia, Plovdiv \\ Branch \\ Department of Transport and Aircraft \\ Equipment and Technologies \\ Plovdiv, Bulgaria \\ e-mail: s.taneva@tu-plovdiv.bg
}

\author{
Krasimir Ambarev \\ Technical University - Sofia, Plovdiv \\ Branch \\ Department of Transport and Aircraft \\ Equipment and Technologies \\ Plovdiv, Bulgaria \\ e-mail: kambarev@tu-plovdiv.bg
}

\author{
Dimitar Katsov \\ Technical University - Sofia, Plovdiv \\ Branch \\ Department of Transport and Aircraft \\ Equipment and Technologies \\ Plovdiv, Bulgaria \\ e-mail: katsov@abv.bg
}

\begin{abstract}
In this paper an interactive system for the study of the movement of a car in a turn is proposed. The interactive system is implemented on a modular principle in the MATLAB environment. The system operates on an algorithm, taking into account the current redistribution of the vertical wheel load and its effect on the characteristics of the lateral slip of wheels with low and ultra-low profile tires. The capabilities of the interactive system are visualized with graphical dependencies.
\end{abstract} tires.

Keywords—algorithm, slip, car, low and ultra-low profile

\section{INTRODUCTION}

Movement of cars with pneumatic tires in turns has been the subject of many studies, following the discovery of Broucherit of the lateral slip of elastic wheels phenomenon in 1925 [1]-[6]. The literature states that when moving through a turn with recording the lateral slip, the centre of the turn shifts [1], [2], [6] and [4]. The analysis of the slip of the front $\delta_{1}$ and rear $\delta_{2}$ axles in the theory [2] determines three types of automobiles: with a normal inclination to turn $\delta_{1}=\delta_{2}$; with a lowered inclination to turn $\delta_{1}>\delta_{2}$, and with increased inclination to turn $\delta_{1}<\delta_{2}$.

It is known that lateral slip characteristics are determined for one wheel with a pneumatic tire on tire testing machines with different level of complexity [7]. When driving in a turn, the wheels of the car slip in different ways due to the redistribution of their vertical load in the centre of the turn.

The purpose of the present work is to propose an interactive programming system in the MATLAB environment to study the analytically the motion of the cars with low and ultra-low profile tires through a turn, taking into account the redistribution of load on the characteristics of the lateral slip of tires. The interactive system allows a dialogue operational mode with the user and allow for quick and accurate calculation.

The interactive system allows as early as the design stage of the car to determine its inclination to turn with the selected tires.

\section{General Regulations}

2.1. Analysis of the movement of a car with low and ultra-low profile tires in a turn

Fig. 1 (a) presents the motion scheme of a $4 \times 2$ car at settled turn with pneumatic wheels slip in a fixed actual centre of the turn p. $\hat{I}_{\delta}$. For a car with hard wheels the centre of the turn is $\hat{I}_{1}$. From Fig. 1 (a) the radius of the turn can be presented as

$$
R_{\delta}=\frac{L}{\operatorname{tg}\left(\theta_{s r}-\delta_{1}\right)+\operatorname{tg} \delta_{2}}
$$

where $\delta_{1}$ and $\delta_{2}$ are respectively the angles of slip of the front and rear axles;

\section{$L$ - wheelbase;}

$\theta_{s r}$ - the average angle of the steered wheels.

The distance from the centre of the turn $\mathrm{p} . \mathrm{O}_{\delta}$ to the centre of gravity of the vehicle p. $C$, according to Fig. 1 (a) can be recorded as

$$
R_{c}=\sqrt{R_{\delta}^{2}-\left(b-R_{\delta} \operatorname{tg} \delta_{2}\right)^{2}}
$$

where $b$ is the longitudinal coordinate of the centre of gravity of the vehicle.

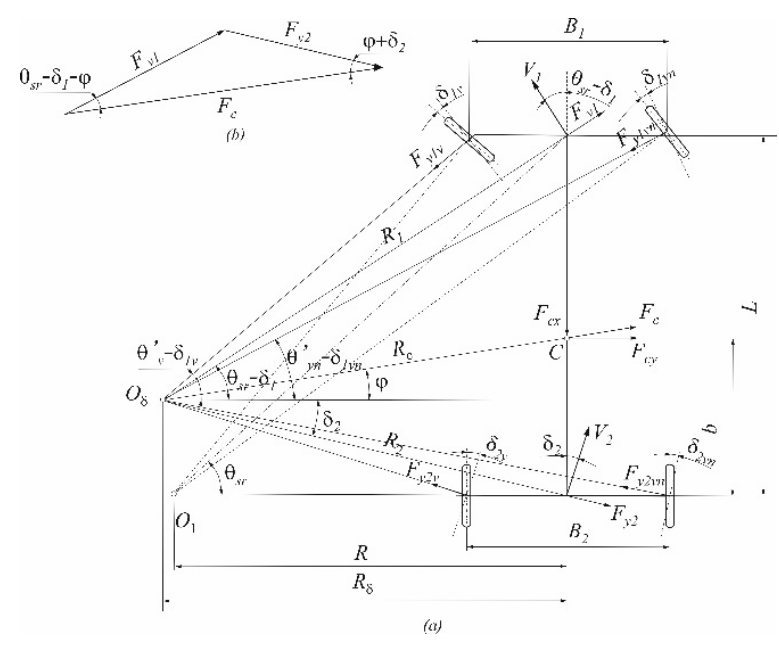

Fig. 1. Scheme of a car in a turn.

From the scheme of Fig. $1(a)$ the actual centre of turn

Print ISSN 1691-5402

Online ISSN 2256-070X

http://dx.doi.org/10.17770/etr2019vol3.4198

(C) 2019 Stiliyana Taneva, Krasimir Ambarev, Dimitar Katsov. Published by Rezekne Academy of Technologies.

This is an open access article under the Creative Commons Attribution 4.0 International License. 
p. $\hat{I}_{\delta}$ determines the lateral slip of the vehicle tires with the following dependencies

$$
\begin{aligned}
& \delta_{1 \mathrm{v}}=\theta_{v}^{\prime}-\operatorname{arctg} \frac{R_{\delta} \operatorname{tg}\left(\theta_{s r}-\delta_{1}\right)}{R_{\delta}-\frac{B_{1}}{2}} \\
& \delta_{1 v n}=\theta_{v n}^{\prime}-\operatorname{arctg} \frac{R_{\delta} \operatorname{tg}\left(\theta_{s r}-\delta_{1}\right)}{R_{\delta}+\frac{\hat{A}_{1}}{2}} \\
& \delta_{2 v}=\operatorname{arctg} \frac{\operatorname{tg} \delta_{2}}{1-\frac{B_{2}}{2 R_{\delta}}} \\
& \delta_{2 v n}=\operatorname{arctg} \frac{\operatorname{tg} \delta_{2}}{1+\frac{B_{2}}{2 R_{\delta}}},
\end{aligned}
$$

where $\hat{A}_{1}, \hat{A}_{2}$ are respectively the track widths of the front and rear axles of the vehicle;

$\delta_{1 v}, \delta_{1 v n}$ - the angles of slip, respectively, of the inner and outer front wheels;

$\delta_{2 v}, \delta_{2 v n}$ - the angles of slip, respectively, of the inner and outer rear wheels.

To determine the reactions on the road, it is necessary to know some of the parameters of the vehicle: vehicle weight $G$, track widths $\hat{A}_{1}, \hat{A}_{2}$, wheelbase $L$, the angles of rotation of the wheels around the steering axis $-\theta_{v}$ $\theta_{v n}$.

For the centrifugal force applied at the centre of gravity of the vehicle, p. $C$, Fig. 1 (a), we obtain the following dependency

$$
F_{c}=\frac{G V_{2}^{2}}{g R_{\delta}^{2}} R_{c} \cos ^{2} \delta_{2}
$$

where $G$ is the weight of the car;

$g$ - acceleration of gravity;

$V_{2}$ - the speed of the rear axle of the vehicle.

The longitudinal and transverse components of the centrifugal force can be presented as

$$
\begin{aligned}
& F_{c x}=\frac{G V_{2}^{2}}{g R_{\delta}^{2}} b \cos ^{2} \delta_{2}-\frac{G V_{2}^{2}}{2 g R_{\delta}} \sin 2 \delta_{2} \\
& F_{o}=G V_{2}^{2} \frac{\cos ^{2} \delta_{2}}{g R_{\delta}}
\end{aligned}
$$

Taking into account the static load on the wheels and taking into account the additional redistribution of loading and unloading according to Fig. 1 (a) for their normal reactions we obtain:

- $\quad$ for the inner front wheel

$$
F_{z 1 v}=\frac{G \cdot b}{2 L}+A \frac{\cos ^{2} \delta_{2}}{R_{\delta}^{2}}-A \frac{\sin 2 \delta_{2}}{2 b R_{\delta}}-B \frac{\cos ^{2} \delta_{2}}{R_{\delta}}
$$

- $\quad$ for the outer front wheel

$$
F_{z 1 v n}=\frac{G \cdot b}{2 L}+A \frac{\cos ^{2} \delta_{2}}{R_{\delta}^{2}}-A \frac{\sin 2 \delta_{2}}{2 b R_{\delta}}+B \frac{\cos ^{2} \delta_{2}}{R_{\delta}}
$$

for the inner rear wheel

$$
\begin{aligned}
& F_{z 2 v}=\frac{G(L-b)}{2 L}-A \frac{\cos ^{2} \delta_{2}}{R_{\delta}^{2}}+A \frac{\sin 2 \delta_{2}}{2 b R_{\delta}}- \\
& -C \frac{\cos ^{2} \delta_{2}}{R_{\delta}} \\
& \quad-\quad \text { for the outer rear wheel } \\
& F_{z 2 v n}=\frac{G(L-b)}{2 L}-A \frac{\cos ^{2} \delta_{2}}{R_{\delta}^{2}}+A \frac{\sin 2 \delta_{2}}{2 b R_{\delta}}+ \\
& +C \frac{\cos ^{2} \delta_{2}}{R_{\delta}}
\end{aligned}
$$

where the coefficients A, B and $\mathrm{C}$ are

$$
A=\frac{G \cdot b \cdot h \cdot V_{2}^{2}}{2 g L} ; B=\frac{G \cdot b \cdot h \cdot V_{2}^{2}}{g L B_{1}} ; C=\frac{G \cdot(L-b) h \cdot V_{2}^{2}}{g L B_{2}}(13)
$$

With the sinus theorem and the conversion of trigonometric expressions (Fig. $1(b)$ ) the total lateral forces acting on the front and the rear axle can be presented as

$$
\begin{aligned}
& F_{y 1}=\frac{G b V_{2}^{2} \cos ^{2} \delta_{2}}{g L R_{\delta} \cos \left(\theta_{\tilde{n} \delta}-\delta_{1}\right)} \\
& F_{y 2}=\frac{G(L-b) V_{2}^{2} \cos ^{2} \delta_{2}}{g L R_{\delta}}
\end{aligned}
$$

The proposed interactive system applies an iterative method for determining the centre of the turn of the vehicle p. $O_{\delta}$.

Dependencies (1) - (15) are valid under the assumption that the centre of the turn p. $O_{\delta}$ in Fig. $1(a)$ is known. In fact, to find the centre of the turn, the angles of the slip need to be known, i.e. $\delta_{1}$ and $\delta_{2}$ of the two axles of the vehicle under the given operating conditions which can be determined experimentally directly on the vehicle or by the lateral characteristics of the wheels obtained on the laboratory equipment.

Specifying the centre of the turn p. $O_{\delta}$ is related, both with the determination of the lateral slip $\delta_{1}$ and $\delta_{2}$ of the axles, and the lateral slips of the front and rear wheels. An iterative method is used in this study, initially assuming that the slip of the pneumatic wheels is zero, i.e. $\delta_{1}=\delta_{2}=\delta_{1 v}=\delta_{1 v n}=\delta_{2 v}=\delta_{2 v n}=0$. In this case, the centre of the turn will be in point $\hat{I}_{1}$ (Fig. $1(a)$ ).

Formulas (1) and (2) define the radius of the turn $R_{\delta}$ and distance $R_{c}$, after which the normal load on the wheels of the vehicle is determined. Using the K. Enke graphical method [9] the characteristics of the lateral slip for the inner wheels are drawn, and the dependencies for the outer wheels are oppositely drawn at the values obtained for the normal wheel load $F_{z 1 v}, F_{z 1 v n}, F_{z 2 v}$, $F_{z 2 v n}$. The charts are positioned so that the axes of the lateral forces $F_{y}$ to coincide and are oppositely directed, and the ordinate axes on which the lateral slip $\delta_{1 v}, \delta_{1 v n}$ of 
the wheels are recorded, are displaced at a distance equal to the lateral force of the front $F_{y 1}$ and the rear $F_{y 2}$ axle. Characteristics for $\delta_{v}=f\left(F_{y v}\right)$ and $\delta_{v n}=f\left(F_{y v n}\right)$ have a mirror image. The ordinate of the intersection of the characteristics of the lateral slip is the common angle of the slip of the front $\delta_{1}$ and the rear $\delta_{2}$ axle (Fig. 2).

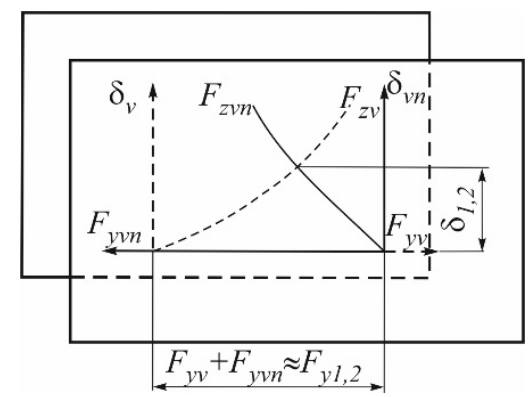

Fig. 2. Determining the lateral slip of the two axles by a mirror image of the lateral forces for the outer and inner wheels displaced at a distance equal to, respectively, the lateral forces of the front and rear axles.

The lack of experimental data on the characteristics of tire slip for normal loads of pneumatic wheels $\left(F_{z 1}, F_{z 2}\right.$ ..... $\left.F_{z n}\right)$, which are obtained when moving in a turn in the range $R_{\min }<R_{\delta}<\infty$, makes the graphical method in this form, inapplicable for studying the turn.

In this work a software was been developed for the method of K. Enke in specifying the radius of the turn $R_{\delta}$ with the ever changing characteristics of the lateral slip of the front and rear wheels $\delta_{1 v y} \overline{\bar{v}} f\left(F_{1}\right)$ $, \delta_{1 v n y} \overline{\bar{v} n} f\left(F_{1}\right), \delta_{2 v y}=f\left(F_{2}\right), \delta_{2 v n y} \overline{\bar{v}} n f\left(F_{2}\right)$.

Through the mirror images of the slip of the outer wheels, the slips of the front $\delta_{1}$ and the rear $\delta_{2}$ axles are determined.

In software modules, non-linear dependencies $F_{y i}=f\left(\delta_{i}\right)$ for all wheels with the corresponding normal load on each wheel are determined by the proposed in [7], [8] dependencies

$$
\begin{gathered}
F_{y i}=k_{t} k_{r} \frac{\operatorname{arctg}\left[\pi k_{\delta 0 i} \delta_{i} /\left(2 \varphi_{y i} F_{z i}\right)\right]}{\pi /\left(2 \varphi_{y i} F_{z i}\right)} \\
F_{y i}=k_{t} k_{r} \frac{\operatorname{arctg}\left[\pi k_{\delta 0 i}\left(\delta_{i}-0,026\right) /\left(2 \varphi_{y i} F_{z i}\right)\right]}{\pi\left(\delta_{i}-0,026\right) /\left(2 \varphi_{y i} F_{z i}\right)} \delta_{i},
\end{gathered}
$$

where $k_{t}$ is the coefficient for taking into account the profile of the tire;

$k_{r}$ - the coefficient measuring the wheel rim size;

$F_{z i}$ - load on $i^{\text {th }}$ wheel in $\mathrm{N}$;

$k_{\delta 0 i}$ - coefficient of resistance against lateral slip of $i^{\text {th }}$ wheel [7], [8];

$\varphi_{y i}$ - grip coefficient of lateral direction of $i^{\text {th }}$ wheel [7], [8].

From experimental studies in [8] to determine of the coefficient of resistance against the lateral slip $k_{\ddot{a} 0 i}$ the averaged experimental curves are used for: the referred length of the contact patch $a_{p r}^{\prime \prime}$, angular stiffness $\tilde{n}_{\omega}^{\prime \prime}$ and grip coefficient of lateral direction $\varphi_{y}^{\prime \prime}$ for low and ultra-low profile tires with rim size from 13 " to 16 ". The coefficient $k_{\delta 0 i}$ is calculated by the dependency $k_{\delta 0 i}=2 c_{\omega}^{\prime \prime} / a_{p r}^{\prime \prime}$, and the grip coefficient of lateral direction by $\varphi_{y i}=k_{\varphi y}^{\prime \prime} \varphi_{y}[8]$.

With the obtained values of the slip of the controllable axle $\delta_{1}$ and the slip of the rear axle $\delta_{2}$ by formula (1) we calculate the new value of the radius of the turn $R_{\delta}$ , while by formula (2) - the distance $R_{c}$. If the deviation of the new $R_{c}^{\text {new }}$ from the old $R_{c}^{\text {old }}$ value of the distance $R_{c}$ is small, the calculation can be stopped. Deviation is determined by the inequality

$$
\left|100\left(\frac{R_{c}^{\text {new }}}{R_{c}^{\text {old }}}-1\right)\right|<\Delta,
$$

where $\Delta$ is the set accuracy of approximation in $\%$. In this study $\Delta=0,1 \%$.

If inequality (18) is not satisfied with the received values of the lateral slip of the front axle $\delta_{1}$ and the rear axle $\delta_{2}$ and the radius $R_{\delta}$ the new wheel loads are determined $F_{z 1 v}, F_{z 1 v n}, F_{z 2 v}, F_{z 2 v n}$ and the lateral forces of the front $F_{y 1}$ and the rear $F_{y 2}$ axle.

Once the inequality is achieved, the actual radius of the turn is specified $R_{\delta}$ and the slip of the front $\delta_{1}$ and the rear $\delta_{2}$ axle.

Formulas (3) and (4) are used to determine the difference $\Delta_{1}$ between the angles of slip of the inner $\delta_{1 v}$ and outer $\delta_{1 v n}$ on the front axle by the dependency

$$
\begin{aligned}
& \Delta_{1}=\delta_{1 v}-\delta_{1 v n}=\left[\theta_{v}^{\prime}-\operatorname{arctg} \frac{R_{\delta} \operatorname{tg}\left(\theta_{s r}-\delta_{1}\right)}{R_{\delta}-\frac{\hat{A}_{1}}{2}}\right]- \\
& -\left[\theta_{v n}^{\prime}-\operatorname{arctg} \frac{R_{\delta} \operatorname{tg}\left(\theta_{s r}-\delta_{1}\right)}{R_{\delta}+\frac{\hat{A}_{1}}{2}}\right]
\end{aligned}
$$

Formulas (5) and (6) are used to define the difference $\Delta_{2}$ between the angles of slip of the inner $\delta_{2 v}$ and outer $\delta_{2 v n}$ wheel on the rear axle by dependency

$$
\Delta_{2}=\delta_{2 v}-\delta_{2 v n}=\operatorname{arctg} \frac{\operatorname{tg} \delta_{2}}{1-\frac{B_{2}}{2 R_{\delta}}}-\operatorname{arctg} \frac{\operatorname{tg} \delta_{2}}{1+\frac{B_{2}}{2 R_{\delta}}}
$$

With the latter (after inequality (18) is achieved) values of the normal wheel load $F_{z i}$, the lateral forces of the front $F_{y 1}$ and the rear axle $F_{y 2}$, the displaced lateral slip characteristics are drawn by the K. Enke method (Fig. 3), by drawing also the characteristics of Fig. 2, but 
displaced at a distance $\Delta_{1,2}$.

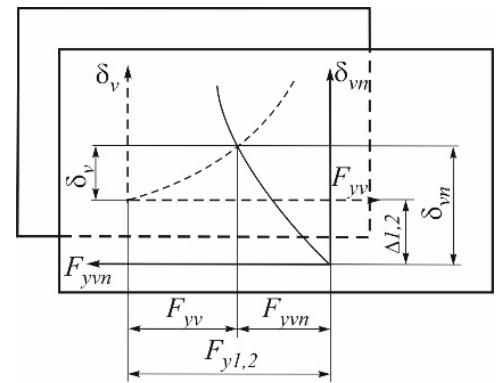

Fig. 3. Determining the lateral reactions and lateral slip of the wheels of the two axles.

The interactive system allows to explore the movement in the turn of $4 \times 2$ and $4 \times 4$ cars.

2.2. Structure of the interactive system

Based on the analytical dependencies presented above and the iterative method (dependency (18)) a program was developed in the MATLAB environment. The blockscheme of the interactive system is presented on Fig. 4. It also has a number of logical operations required by the specifics of the calculations performed. The programme also uses the obtained experimental results for drawing dependencies $F_{y i}=f\left(\delta_{i}\right)$ [7], [8]. The interactive system allows graphic analysis determination of the slip of the inner $\delta_{1 v}, \delta_{2 v}$ and the outer $\delta_{1 v n}, \delta_{2 v n}$ wheels of the car and the lateral slip in a turn of the front $\delta_{1}$ and the rear $\delta_{2}$ axle. All this allows for automated analytical study of the movement in a turn of cars with low and ultra-low profile tires, including the impact of varying initial data on vehicle stability and handling.

The mass and geometric parameters of the vehicle are initially introduced. The initial data are: the mass of the vehicle $m$, the track width of the front $\hat{A}_{1}$ and the rear $\hat{A}_{2}$ axle, the wheelbase $L$, the coordinates of the mass centre of the car $b, h$, the angles of rotation of the inner $\theta_{v}$ and outer $\theta_{v n}$ wheels and the tire dimensions $-B_{t}, H_{t}, d_{r}$.

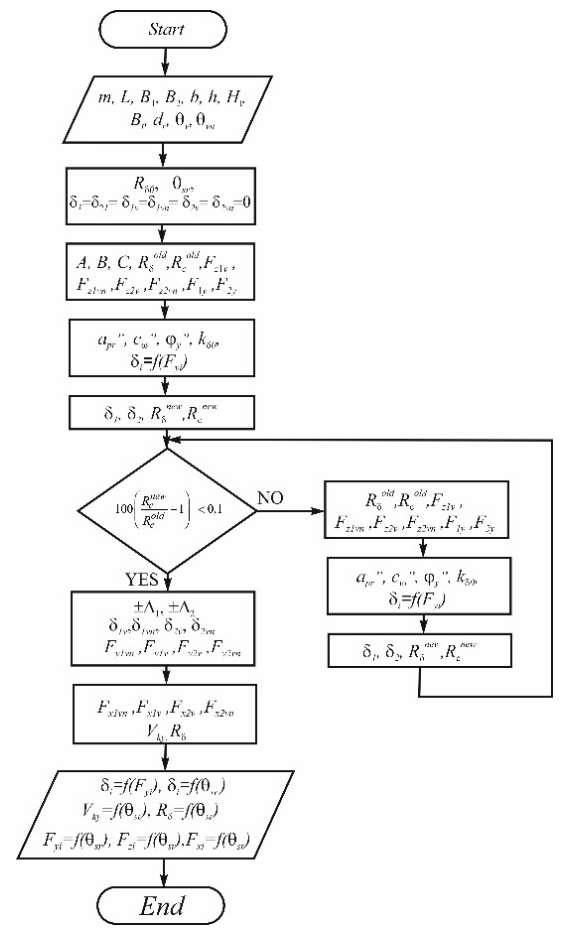

Fig. 2. Block-scheme of the interactive system.

\subsection{Results}

For visualizing the capabilities of the interactive system, the initial data of a car Honda Civic Aerodeck 1.5iLS were used with tire dimensions $185 / 60 \mathrm{R} 14$. The graphical dependencies shown in Fig. 5 and Fig. 6 are at a given vehicle speed $V=20 \mathrm{~km} / \mathrm{h}$, vertical wheel loads, respectively $F_{z 1 v}=3,164 \mathrm{kN}, \quad F_{z 1 v n}=4,886 \mathrm{kN}$, $F_{z 2 v}=3,175 \mathrm{kN}, F_{z 2 v n}=4,962 \mathrm{kN}$ and air pressure in the tires $p_{b}=0,25 \mathrm{MPa}$. Fig. 5 illustrates the graphical dependencies of the characteristics of slip of the front $\delta_{1 v n}=f\left(F_{y 1 v}\right), \delta_{1 v n}=f\left(F_{y 1 v n}\right)$ and the rear $\delta_{2 v}=f\left(F_{y 2 v}\right), \quad \delta_{2 v n}=f\left(F_{y 2 v n}\right)$ wheels, and Fig. 6 shows the changes of the slips of the front $\delta_{1}$ and the rear $\delta_{2}$ axles from the average angle of the steered wheels $\theta_{s r}=15^{\circ}$ of the car.

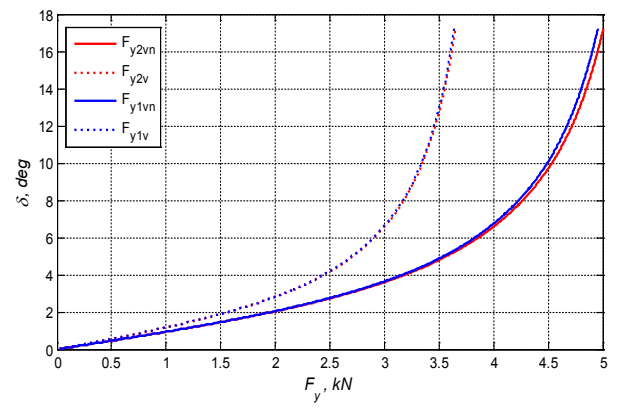

Fig. 3. Characteristics of lateral slip of the car's inner and outer tires.

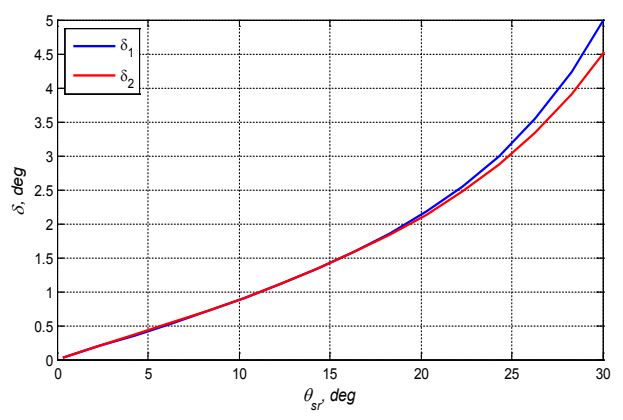

Fig. 4. Lateral slip of the front $\delta_{1}$ and the rear $\delta_{2}$ car axle.

III. CONCLISIONS

From the results for the slip of the front $\delta_{1}$ and rear $\delta_{2}$ axle, as well as the angles of slip of the outer $\delta_{1 v n}, \delta_{2 v n}$ and inner $\delta_{1 v}, \delta_{2 v}$ wheels under the above conditions, the following conclusions can be drawn:

- The Honda Civic Aerodeck car has a reduced inclination to a turn for the entire steered wheel rotation range.

- The characteristics of the lateral slip of the inner $\delta_{1 v}, \delta_{2 v}$ and the outer $\delta_{1 v n}, \delta_{2 v n}$ wheels are approximately equal which is due to the centre of gravity position in the centre of the car.

- The proposed interactive system for studying the turn of a passenger car with low and ultra-low profiled tires allows to quickly and accurately examine the kinematic and dynamic parameters of the car's wheels as early as the design stage of the vehicle. 


\section{ACKNOWLEDGEMENTS}

The authors would like to thank the Research and Development Sector at the Technical University of Sofia for the financial support.

Our research, the results of which are presented in this article have been carried out under the Programme Young Scientists and Postdoctoral Students of the Ministry of Education and Science, Bulgaria.

\section{REFERENCES}

[1] Litvinov A. S, Handling and stability of the car. M., Mechanical Engineering, 1971.

[2] Lyubenov S, Angelov B, Evtimov I, Vehicles and Tractors. Performance properties, Ruse, 2004.

[3] Pacejka H. B, Tyre and Vehicle Dynamics. SAE, Warrendale, 2002.

[4] Pacejka H. B, Tyre and Vehicle Dynamics. Second edition, Amsterdam, Boston, Heidelberg, London, New York, Oxford, Paris, San Diego, San Francisco, Singapore, Sydney, Tokyo, ISBN -13: 980-0-7506-6918-4, 2006.
[5] Katsov D. A., Hlebarski D. A, Dynamics of the turn of wheel vehicle with account of the deformations of the tires and the influence of the suspension. International Journal of Computational and Numerical Analysis and Applications, Vol. 6, No. 2, pp.97106, 2004, ISSN 1311-6789.

[6] Katsov D. A., Hlebarski D. A, Kinematics of the turn of wheel vehicle with account of the deformations of the tires and the influence of the suspension. International Journal of Computational and Numerical Analysis and Applications, Vol 25, No. 2, pp. 203-214, 2005, ISSN 1311-8080.

[7] Katsov D., S. Taneva, On obtaining the lateral slip characteristics of low and superlow profile tires of cars. Journal of Machine Building and Machine Science, Varna, Bulgaria, year. X, No. 2 , vol. 24, pp. 33-38, 2015, ISSN 1312-8612.

[8] Katsov D., S. Taneva, Investigation of the lateral slip and grip of low and superlow profile tires of cars. Journal of Machine Building and Machine Science, Varna, Bulgaria, year. X, No. 2, vol. 24, pp. 39-45, 2015, ISSN 1312-8612.

[9] Katsov D.A, Study on stabilization of the steered wheels of a wheeled tractor $4 \times 2$ - class $14 \mathrm{kN}$. PhD thesis, "Angel Kanchev" University of Ruse, Bulgaria, 1987. 